\title{
Assessment of Knowledge, Attitude and Practices Towards Onchocerciasis Prevention and Control Among Residents of Kometa Kebele, Bench Maji Zone, South Western Ethiopia
}

\author{
Beteab Getachew ${ }^{1} \quad$ Abiyu Tadele ${ }^{2 \#}$ \\ 1.Gebretsadik Shawo General Hospital, Bonga, Ethiopia \\ 2.Department of Animal Science, Bonga University, Ethiopia
}

\begin{abstract}
Onchocerciasis is one of the most important public health problems over large areas of tropical African countries including Ethiopia. Involvement of individuals and communities are an important component of Onchocerciasis prevention and control methods. The objective of this study was to assess communities knowledge, attitude and practices towards prevention and control of Onchocerciasis in Kometa Kebele. A cross-sectional study was carried out in Kometa kebele in which 362 respondents who were above 18 years of age were considered in data collection. Data were collected using a pre-tested questioner administered to sample respondents which was then analyzed using SPSS version 20. The results indicated that majority of the respondent were male (55\%) and $32.9 \%$ of the interviewed respondents attended primary school. The average family size per household was 5.5 person. Majority of the respondents (64.4\%) knew about the disease Onchocerciasis. About $36.5 \%$ of respondents mentioned Onchocerciasis can be transmitted, of which $50.8 \%$ knew that the transmission is related to black fly biting. Majorities (90.3\%) of the respondents mentioned that onchocerciasis is treatable disease with drug. In the present study $85.9 \%$ of the study respondents perceived that, Onchocerciasis could cause blindness and death. In the present study $79.6 \%$ of respondents did not wear protective clothes when they are working around fast flowing water. In the study area more than 82 percent of the respondents take ivermectin for Onchocerciasis prevention. About 67,64 and $60.4 \%$ of respondents had good knowledge, attitude and practices, respectively. In conclusion, most of the respondents knew the disease Onchocerciasis, however they majorities lack awareness on the causative agent, mode of transmission and prevention of Onchocerciasis. Thus, it is important to create awareness and implement intervention activities among communities about Onchocerciasis in the area to prevent and control the disease and achieve the desired goal at national level.
\end{abstract}

Keywords; Attitude, Ethiopia, Knowledge, Kometa, Onchocerciasis, Practice.

DOI: $10.7176 / \mathrm{JHMN} / 96-02$

Publication date: December $31^{\text {st }} 2021$

\section{INTRODUCTION}

Onchocerciasis, commonly known as 'river blindness', is a debilitating vector-borne disease which is mainly caused by a parasite, Onchocerca volvulus (Daniel et al., 2015). It could be transmitted by the bite of black-fly, Simulium damnosum, which can breeds in fast flowing streams and rivers (Fitsum et al., 2016). The disease is also termed river blindness because the vector, the blackfly, is commonly found in areas where there is a rapidly flowing streams and rivers. The common manifestation of the infection is blindness. The disease is also characterized by causing skin lesions with severe itching, a serious eye lesion and blindness known as river blindness. It is a chronic and slowly progressive disease. The initial infestation often occurs in childhood, and many of the affected individuals remain asymptomatic for long periods (Fitsum et al., 2016)

Onchocerciasis is among the most important public health problems which is widely distributed over 30 tropical country and in six countries of Latin and Central America and one Arabian Peninsula (Fitsum et al., 2016). Around 125 million people in the world are estimated at risk of the infection and majorities of them are found in Africa (Wogu and Okaka, 2008). In Ethiopia, 3 million people are already infected, whereas 7.3 million are at risk of infection and almost everyone in an endemic village will harbor the disease. Nine regions surveyed for river blindness were shown to be endemic; the endemic areas extend from the northwest part to South West part of the country that borders Sudan (Rasheed, 2007). Mass treatment of high risk communities with ivermectin was adopted in line with the African Program for Onchocerciasis Control (APOC) community directed treatment with ivermectin (CDTI) strategy (WHO, 2000, 2010). The Right to Sight global initiative was launched in 1999 by the World Health Organization (WHO) and The International Agency for the Prevention of Blindness (IAPB), with the aim of eliminating avoidable blindness by the year 2020 and in so doing preventing an estimated 100 million people from going blind. An indicator of the impact of VISION 2020 is whether the prevalence of avoidable blindness is indeed declining (FDRE, 2016). The main control strategy for onchocerciasis in Ethiopia is mass treatment with ivermectin (Wogu and Okaka, 2008). The ultimate goal of the APOC is to reduce the public health and socio-economic problems of onchocerciasis by providing the administration of the tablet for a period of $12-15$ years using the strategy of yearly CDTI in endemic areas to kill 
the microfilariae that invade the eyes and are present in the skin to be transported to another victim by the black fly. Ivermectin drug is highly effective in the mass treatment of onchocerciasis, it kill the microfilariae that invade the eyes and are present in the skin to be transported to another victim by the black fly.

Onchocerciasis is one of the major causes of morbidity in South West part of Ethiopia, the result studied by various scholars in the country indicated that, nearly one and half million cases of Onchocerciasis are estimated to exist in Ethiopia, especially in South West in an area of about 300,878 square kilometers; the population at risk of infection is estimated to be 7.3 million. The disease affects population in habituating the fertile area of the country and discourages development schemes due to decrease force and decline individual productivities which result increase of dependency and poverty (Fitsum et al., 2016). In Sheka Zone, the study carried out in 1993 indicated that, the existence of Onchocerciasis in this particular area and given an opportunity as a beginning of ivermectin drug distribution for treatment of Onchocerciasis (Workneh et al., 1993). Due to the existence of large volume of rivers and streams flowing throughout the year in Bench Maji Zone and the adjoining Zones, the possibility of disease occurrence might be high. Since, Bench Maji Zone is situated adjacent to Sheka Zone, after the identification and distribution of ivermectin drug for treatment the community's knowledge, attitude and practices towards prevention and control of Onchocerciasis in Bench Maji Zone, Kometa kebele was not studied and yet known. Therefore, the aim of this study was to investigate the communities knowledge, attitude and practice towards onchocerciasis in Kometa Kebele South Western Ethiopia.

\section{Significance of the Study}

Onchocerciasis is one of the major causes of morbidity in South West part of Ethiopia including the study area. Therefore, assessment of KAP of the kebele residents towards prevention and control of Onchocerciasis could:-

$>$ Help health planners and health educators providing basic information to focus on its Prevention and control.

$>$ Provide a baseline data on the awareness and practice of Onchocerciasis prevention for the study area.

$>$ Use as base line data for further researches who want to conduct on prevention and control of Onchocerciasis.

$>$ Moreover, the finding used directly or indirectly concerned bodies to plan, control and prevent the critical problems of Onchocerciasis.

\section{Objectives}

General Objective

* To assess, communities knowledge, attitude and practices towards prevention and control of Onchocerciasis in Mizan Aman town Kometa kebele, South Western Ethiopia.

\section{Specific Objectives}

$>$ To assess communities knowledge about prevention and control of Onchocerciasis among respondents of Kometa kebele.

$>$ To assess communities attitude towards prevention and control of Onchocerciasis among respondents of Kometa kebele.

$>$ To assess communities practice on prevention and control of Onchocerciasis among respondents of Kometa Kebele.

\section{METHODOLOGY}

\section{Description of the study area}

This study was conducted in Aman town Kometa kebele, B/Maji Zone, SNNPR, Ethiopia. Kometa kebele is found in South-Western part of Ethiopia, which is $565 \mathrm{~km}$ away from the capital city of Ethiopia (AA). The total number of households found in Kometa kebele were 2,221; Kometa kebele is situated $3 \mathrm{~km}$ away from Mizan Tepi Teaching hospital. The main ethnic groups are Bench, Kaffa and Amhara. The major religions are Orthodox, Protestant and Muslim. The official working language is Amharic (AK, 2017).

\section{Study design}

Community based cross-sectional study design was used that employ qualitative and quantitative data collection method.

\section{Source population}

Representative households from Kometa kebele were source population

\section{Study population}

All respondents aged above 18 years were considered as source population. 


\section{Sample size determination}

Using a single proportion cross sectional formula, the sample size calculation consider the following assumptions;- Since there is no research done in study area previously, estimate of proportion --- 50\% Degree of accuracy------ 5\% Confidence level --- 95\%

Sample was size Calculated --- $n=Z^{2} p(1-p) / w^{2}=(1.96)^{2}(0.5 \times 0.5) /(0.05)^{2}=\underline{385}$

However, the total number of households which are found in Kometa kebele were (N) 2221; Since it is less than 10,000, adjustment was made using correction formula;- $\mathrm{n} /(1+(\mathrm{n} / \mathrm{N})) 385 /(1+(385 / 2221))=328.12$; approximately $=\underline{\mathbf{3 2 9}}$

Using Non response rate of $10 \%(329 * 10 / 100=33)$, the sample size became 362 . Thus, a total of 362 respondents were involved for this study.

\section{Sampling technique}

The study participants/respondents were selected by using systematic sampling technique and the first household was selected using lottery method. When calculated the interval $\mathrm{K} /$ or the sampling fraction become: $\mathrm{n} / \mathrm{N}=$ $(2221 / 362)$ which was approximately equal to $1 / 6$; which is in every $6^{\text {th }}$ house the data collection process was made until the desired sample size was achieved.

\section{Method of data procedure}

Data collection tools (questioner) was adapted after review of relevant literatures. The questionnaire and statement was grouped and arranged according to the particular objectives that they could address.

Data was collected face to face interview method with in the household's house to house visit using structured questionnaire. Data collectors were enumerated and trained for one day about objectives, questionnaire and interview techniques before they go to data collection. The principal investigators were closely supervising and also collected data. Study Variables are Knowledge, Attitude and Practices.

\section{Operational Definitions}

Good knowledge- Those respondents who are able to score $\geq 70 \%$ of the total knowledge questions on Onchocerciasis prevention and control; Poor knowledge- Answering $<60 \%$ of the knowledge question on prevention and control of Onchocerciasis; Positive attitude- Those respondents who are able to score $\geq 70 \%$ of the total attitude questions on Onchocerciasis prevention and control; Negative attitude- Those respondents who are able to score $\leq 60 \%$ of the total attitude question on Onchocerciasis prevention and control; Good practiceThose respondents who are able to score $\geq 70 \%$ of the total practice questions on Onchocerciasis prevention and control and Poor practice- Those respondents who are able to score $\leq 60 \%$ of the total practice questions on Onchocerciasis prevention and control.

\section{Data Processing and Analysis}

Collected data were checked for completeness, missing value and complied on excel 2007 and analyzed using SPSS version 20. The result of study was summarized using tables, figures and presented in frequency and percentage.

\section{Results}

Socio-demographic characteristics of respondents in the study area

In the present study majority of the study participants were male $(55 \%)$ in which the mean age was 32.6 year. Regarding their marital status, majority $(74.9 \%)$ were married, $(13.8 \%)$ were divorced and $(11.3 \%)$ were single. The predominant religions of the study participants was Orthodox and Protestant (43.4 and 36.2) percent, respectively. The educational status of the study participants indicates primary school (32.9\%), secondary school (26.8\%) and illiterate (21.8). 
Table 1. Socio-demographic characteristics of the respondents in the study area

\begin{tabular}{lcc}
\hline Household characteristics & Kometa Kebele \\
\cline { 2 - 3 } Sex of respondents & $\mathbf{N}$ & \% \\
$\quad$ Male & & 55 \\
$\quad$ Female & 199 & 45 \\
Marital status & 163 & 11.3 \\
$\quad$ Single & & 74.9 \\
Married & 41 & 13.8 \\
$\quad$ Divorced & 271 & 21.8 \\
Educational level & 50 & 32.9 \\
Illiterate & & 26.8 \\
$\quad$ Primary School & 79 & 18.5 \\
Secondary school & 119 & 43.4 \\
College and above & 97 & 36.2 \\
Religious status & 67 & 20.4 \\
$\quad$ Orthodox & & 157 \\
$\quad$ Protestant & 131 & 74 \\
$\quad$ Muslim & & \\
\hline
\end{tabular}

N, Number of observations

Respondents knowledge on prevention and control of Onchocerciasis in the study area In the present study indicated in (Table 2), most of the respondents knew about the causes of onchocerciasis by stating black flies as the main cause (43.4\%), however majorities (48.9\%) still did not know the causes of onchocerciasis in the study area.

Table 2. The respondents response on the causes of onchocerciasis in the study area

\begin{tabular}{lll}
\hline Causes of Onchocerciasis & $\mathbf{N}$ & $\mathbf{\%}$ \\
\hline Scorching of the sun & 3 & 0.8 \\
Hereditary & 5 & 1.4 \\
Black flies bite & 157 & 43.4 \\
Mosquitoes' bite & 13 & 3.6 \\
Parasite & 7 & 1.9 \\
Don't know & 177 & 48.9 \\
\hline
\end{tabular}

$N$, Number of observations

In the present study, majority of the respondents (64.4\%) knew about the disease Onchocerciasis, about $36.5 \%$ of respondents mentioned onchocerciasis can be transmitted, of which $50.8 \%$ knew that the transmission is related to black fly biting. The rest of $49.2 \%$ had misconceptions about mode of transmission of onchocerciasis. Majorities $(90.3 \%)$ of the respondents mentioned that onchocerciasis is treatable disease with drug (Table 3).

Table 3. Respondents response on knowledge of Onchocerciasis

\begin{tabular}{|c|c|c|c|c|}
\hline \multirow[t]{2}{*}{ Indicative questions on knowledge } & \multicolumn{2}{|c|}{ Yes } & \multicolumn{2}{|c|}{ No } \\
\hline & $\mathbf{N}$ & $\%$ & $\mathbf{N}$ & $\%$ \\
\hline \multicolumn{5}{|l|}{ Do you know the disease Onchocerciasis } \\
\hline & 233 & 64.4 & 129 & 35.6 \\
\hline Can Onchocerciasis be treated with drug & 327 & 90.3 & 35 & 9.7 \\
\hline Do you know black flies & 184 & 50.8 & 178 & 49.2 \\
\hline Does Onchocerciasis transmit from person to person & 132 & 36.5 & 230 & 63.5 \\
\hline
\end{tabular}

\section{$N$, Number of observations}

The result pertaining to the respondents response on the sign and symptoms of onchocerciasis indicated in (Figure, 1), majority of the respondents $(56.08 \%)$ said body itching could be the main sign and symptoms followed by body swelling (22.10\%). 


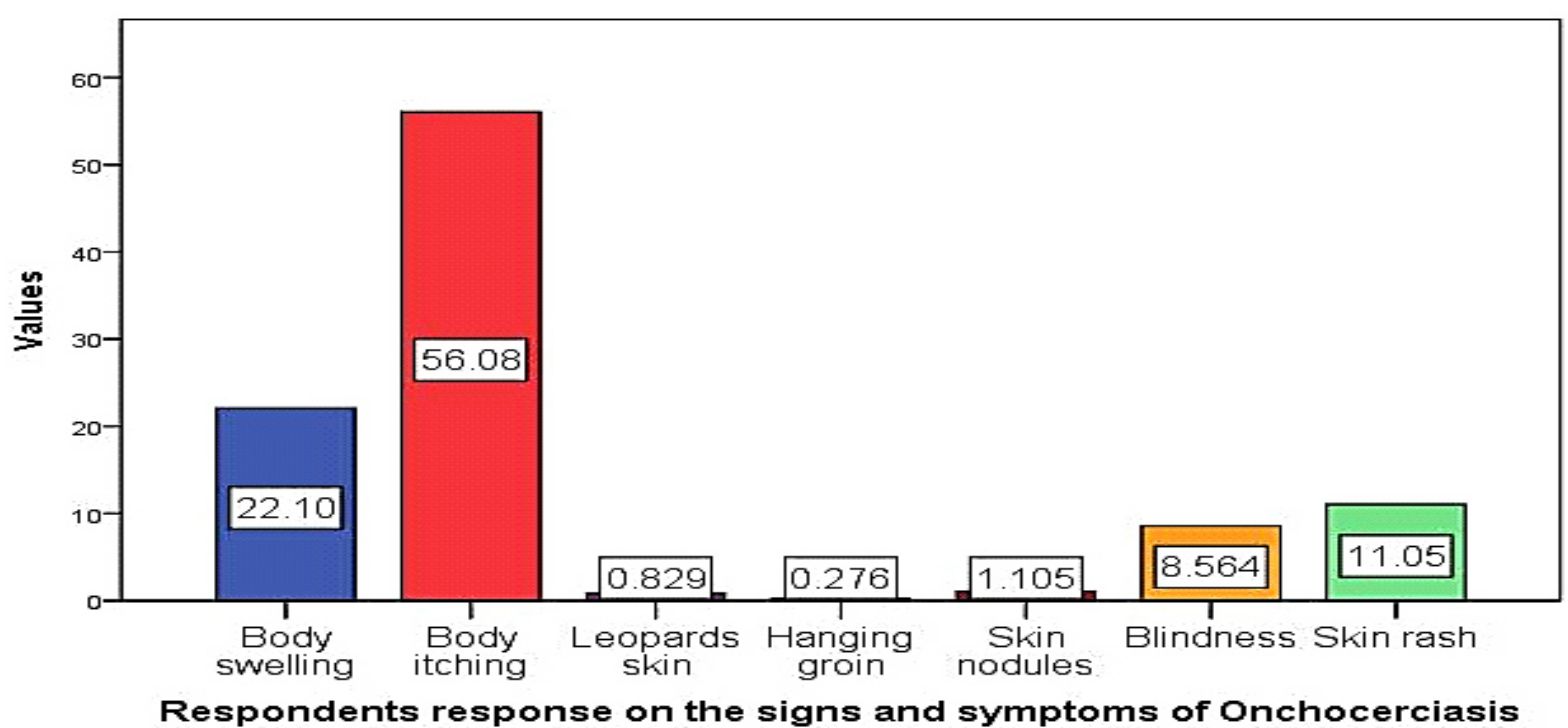

Figure 1. Respondents response on sign and symptoms of Onchocerciasis

\section{Respondents attitude towards prevention and control of Onchocerciasis}

In the present study majority $(85.9 \%)$ of the study respondents perceived that, Onchocerciasis could cause blindness and death. In addition, $72.7 \%$ of respondents think that, treatment with ivermectin drug could bring drug related adverse effects. However, most of the respondents $(61.3 \%)$ consider that, Onchocerciasis is not a health problem of the community. Similarly, 60.5 percent of respondents recognize that, Onchocerciasis does not transmit from ill person to a healthy person. In this study, most of the respondents $(72.7 \%)$, wearing protective clothes cannot prevent from the bite of female black fly (Tale 4).

Table 4 . Respondents attitudes towards prevention \& control of onchocerciasis

\section{Indicative questions}

Is Onchocerciasis a health problem of this community?

Does Onchocerciasis transmit from ill person to a healthy person?

Wearing protective clothes can prevent from the bite of female black fly

Onchocerciasis could cause blindness and death

Ivermectin could bring drug related adverse effects?

\begin{tabular}{cccc}
\multicolumn{2}{c}{ Agree } & \multicolumn{2}{c}{ Disagree } \\
\hline $\mathbf{N}$ & $\mathbf{\%}$ & $\mathbf{N}$ & $\mathbf{\%}$ \\
140 & 38.7 & 222 & 61.3 \\
143 & 39.5 & 219 & 60.5 \\
99 & 27.3 & 263 & 72.7 \\
311 & 85.9 & 51 & 14.1 \\
263 & 72.7 & 99 & 27.3 \\
\hline
\end{tabular}

$N$, Number of observations

In the current study the respondent's attitudes towards prevention and control of Onchocerciasis were indicated in (Table 5). Majorities (63.3\%) indicated that, extension workers are mainly involved in providing the drug. In this study respondents indicated that morning and night (37.6\%) are the most suitable time for the bite of female black fly.

Table 5. Respondents attitudes towards prevention \& control of onchocerciasis

\begin{tabular}{|c|c|c|c|}
\hline Indicative questions & Categories & $\mathbf{N}$ & $\%$ \\
\hline \multicolumn{4}{|c|}{ Who do you think is best for Ivermectin drug provision? } \\
\hline & Community Drug Distributors & 25 & 6.9 \\
\hline & Extension workers & 229 & 63.3 \\
\hline & Kebele managers & 46 & 12.7 \\
\hline & Health professionals & 62 & 17.1 \\
\hline \multicolumn{4}{|c|}{ When do you think that female black fly bite most often? } \\
\hline & Morning & 59 & 16.3 \\
\hline & Night & 86 & 23.8 \\
\hline & Midday & 50 & 13.8 \\
\hline & Morning \& night & 136 & 37.6 \\
\hline & Don`t know & 31 & 8.6 \\
\hline \multicolumn{4}{|c|}{ Do you support or oppose use of ivermectin for prevention? } \\
\hline & Support & 262 & 72.4 \\
\hline & Oppose & 35 & 9.7 \\
\hline & Don't mind & 65 & 18.0 \\
\hline
\end{tabular}

$N$, Number of observations 


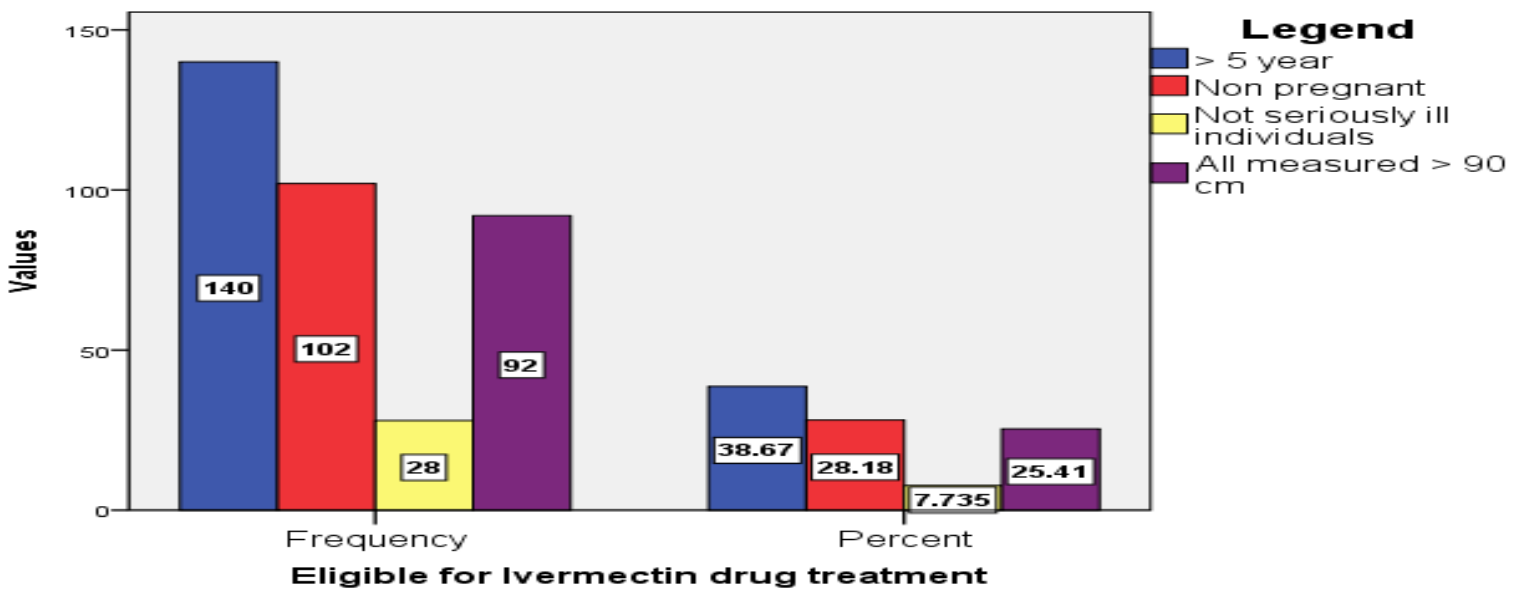

Figure 2. Individuals eligible for ivermectin treatment

In the current study related with the individuals who are eligible for ivermectin drug treatments were indicated in (Figure 2), in which majorities $(38.67 \%$ ) of the study respondents said, those who are greater than five years old are eligible for taking the drug.

\section{Respondents practice towards prevention and control of Onchocerciasis}

In the current study majority $(79.6 \%)$ of respondents did not wear protective clothes/or shoes when they are working around fast flowing water. In the study area more than 82 percent of the respondents take ivermectin for Onchocerciasis prevention. About $58.6 \%$ did not participate during mass treatment with ivermectin. In this study 59.1 percent's said that, there was no black fly breeding site in their surroundings. However, more than 72 percent of the respondents sleep under a treated net (72.9\%), use bed net at night time $(73.5 \%)$ and slept under a treated net during the previous night $(73.2 \%$ ), respectively (Table 6).

Table 6. Respondents practices on prevention and control of onchocerciasis

\begin{tabular}{|c|c|c|c|c|}
\hline \multirow[b]{2}{*}{ Indicative questions on practices } & \multicolumn{2}{|c|}{ Yes } & \multicolumn{2}{|c|}{ No } \\
\hline & $\mathbf{N}$ & $\%$ & $\mathbf{N}$ & $\%$ \\
\hline $\begin{array}{l}\text { Do you wear protective clothes/shoes when you are doing around fast flowing } \\
\text { water? }\end{array}$ & 74 & 20.4 & 288 & 79.6 \\
\hline Are you taking ivermectin for Onchocerciasis prevention? & 298 & 82.3 & 64 & 17.7 \\
\hline Have you ever take ivermectin drug before? & 325 & 89.8 & 37 & 10.2 \\
\hline Do you participate during mass treatment with ivermectin? & 150 & 41.4 & 212 & 58.6 \\
\hline Is there black fly breeding site available nearby your home? & 148 & 40.9 & 214 & 59.1 \\
\hline Have you ever participating in environmental management for black fly control & 177 & 48.9 & 185 & 51.1 \\
\hline Have you ever sleep under a treated net? & 264 & 72.9 & 98 & 27.1 \\
\hline Do you use bed net at bed time? & 266 & 73.5 & 96 & 26.5 \\
\hline Did you sleep under a treated net during the previous night? & 265 & 73.2 & 97 & 26.8 \\
\hline
\end{tabular}

$N$, Number of observations

In the present study the respondents who had good knowledge, positive attitude and practices about onchocerciasis were 67,64 and $60.4 \%$, respectively.

Table 7. Level of knowledge, attitude and practices of respondents on onchocerciasis

\begin{tabular}{lcccc}
\hline \multirow{2}{*}{ Outcome variables } & \multicolumn{2}{c}{ High level } & \multicolumn{2}{c}{ Low level } \\
\cline { 2 - 5 } \multicolumn{1}{c}{} & $\mathrm{N}$ & $\%$ & $\mathrm{~N}$ & $\%$ \\
\hline Knowledge & 243 & 67 & 119 & 33 \\
Attitude & 233 & 64 & 129 & 36 \\
Practices & 218 & 60.4 & 144 & 39.6 \\
\hline
\end{tabular}

$N$, Number of observations

\section{Discussion}

Respondents socio-demographic characteristics in the study area

In the present study the highest percentage of male (55\%) than female (45\%) was observed, which was in a good agreement with the results of various scholars in the country (Dana et al., 2015; Adugna et al., 2015; Fitsum et al., 2016). The result on educational level obtained in the current study indicated majorities (32.9\%) 
were attained primary education, which was also in line with the findings of Fitsum et al. (2016). In this study, both male and female respondents engage in socio-economic activities such as fetching water which can expose them to black fly biting. Formal education appeared to be one of the medium for the awareness of the disease. As a result of the formal education that most of the respondents had, the study showed that $32.9 \%$ of the respondents have already acquired primary education are aware of the disease. Despite the fact that more than half of the respondents know about the disease.

\section{Respondents knowledge, practices and attitude}

In the present study majorities $(48.9 \%)$ of the study participants did not knew the causative agents of Onchocerciasis. In line with this, many authorities believe that low knowledge and practice of the communities in Africa predispose them to infection (Ukoli, 1992). On the other hand, majority (43.4\%) of the respondents associate the causative agent of the disease with the bite of black flies, which is comparable with the finding of study conducted in Bebeka, Southwest Ethiopia (Yirga et al., 2008). Similarly, in this study, majority of the cause with the bite of mosquito and parasite, which is consistent with the findings of the study conducted in Bebeka, Southwest Ethiopia (Fitsum et al., 2016).

In the present study pertaining to sign and symptoms, body itching $(56.08 \%)$ and body swelling $(22.10 \%)$ were the major sign and symptoms. This result was in agreement with the findings of Fitsum et al. (2016) in which body itching was the main symptom. On the other hand, this result was also in the same scenario with the findings of Agbolade et al. (2010) and Adesina et al. (2017) who reported itching to have a high percentage, followed by swelling and itching. In the present study, majorities $(79.6 \%)$ of the respondents did not wear protective cloths to prevent from bite of black fly, even though they work around fast flowing rivers. This finding is in close agreement with the reports of Fitsum et al. (2016). In addition, the communities practices towards prevention of Onchocerciasis were taking ivermectin drug $(82.3 \%)$ and using bed net $(73.5 \%)$, which is in close agreement with the findings of Fitsum et al.(2016).

In this study, majority of the study participants based on the operational definition had poor knowledge of onchocerciasis (i.e. only $67 \%$ of the study participants had good knowledge). Similarly, majority of the study subjects had poor attitude and practice about onchocerciasis (i.e. only 64 and $60.4 \%$ study subjects had good attitude and good practice about onchocerciasis, respectively). This finding is also consistent with the findings of the study conducted in Sequa area, Southwest Ethiopia (Yesuf, 2006); the study conducted in Quara district, Northwest Ethiopia (Fitsum et al., 2016) and the study conducted in Homa District, Western Ethiopia (Wayuma, 2015). This is probably due to shortage of health education at the community level and the CDDs may not be properly trained about onchocerciasis due to negligence of health extension workers to supervise the CDDs in delivering health education, and/or excluding the community interventions for onchocerciasis in the health extension package.

\section{Conclusion and Recommendation}

In conclusion, even though most of the respondents knew the disease Onchocerciasis, majority of them still lack awareness on the causative agent, mode of transmission and prevention of Onchocerciasis. In addition, the respondents associate the causative agent of the disease with the bite of black flies. Some others, associate the cause with the bite of mosquito and parasite. In relation with the sign and symptoms of Onchocerciasis, body itching and body swelling were the main indicatives identified by the respondents. In prevention and control mechanisms, respondents did not wear any protective cloths to prevent from bite of black fly, even though they work around fast flowing rivers. This could predisposes the communities to infection with Onchocerciasis and also interfere with the activities which could be undertaken to prevent and control the disease. Therefore, it is necessary to improve communities' awareness about Onchocerciasis. In addition, hhealth extension workers should properly deliver adequate training on prevention and control of Onchocerciasis for the community. Health service centers should collaborate with concerned professionals in the area to prevent Onchocerciasis.

\section{Conflict of interests}

The authors have not declared any conflict of interests.

\section{Acknowledgements}

The authors would like to acknowledge Mizan Aman office of health center for their provision of information. Finally we are also indebted to thank those households and health extension workers who participated in this study.

\section{References}

Abanobi, O.C., 2000. Community based mass distribution of ivermectin for the control of human onchocerciasis in Ehime communities, Imo State, Nigeria. Eye and Vision, 41-56. 
Adesina F. P., Awosolu O. B., Olusi T. A., 2017. Incidence of Onchocerciasis and Vector Knowledge among Residents of Some Parts of Ondo State, Nigeria. Public Health Research, 7(5): 107-111.

Adugna Endale, Berhanu Erko, Fitsum Weldegebreal and Mengistu Legesse, 2015. Predictors of compliance with community-directed treatment with ivermectin for onchocerciasis control in Kabo area, southwestern Ethiopia, Parasites \& Vectors; 8:99: 1-8.

Agbolade, O.M., Odutola, O. M., Bolatito, I. and Ndubuisi, C. A., 2010. Biting knowledge of blackfly among students and workers of a Nigerian tertiary institution. International Journal of Medicine and Medical Sciences 2(6): 196 - 199.

Daniel Dana, Serkadis Debalke, Zeleke Mekonnen, Wondwossen Kassahun, Sultan Suleman, Kefelegn Getahun and Delenasaw Yewhalaw, 2015. A community-based cross-sectional study of the epidemiology of onchocerciasis in unmapped villages for community directed treatment with ivermectin in Jimma Zone, Southwestern Ethiopia.1-7.

Fitsum Weldegebreal, Girmay Medhin, Zemichael Weldegebriel and Mengistu Legesse, 2016. Knowledge, attitude and practice of community drug distributors' about onchocerciasis and community directed treatment with ivermectin in Quara district, North Western Ethiopia.1-8.

Legesse M, Balcha F, Erko B., 2010. Status of onchocerciasis in Teppi area, Southwestern Ethiopia, after four years of annual community-directed treatment with ivermectin. Ethiop J Health Dev 24: 51-56.

M. D. Wogu and C. E. Okaka, 2008. The knowledge, attitude and perception of onchocerciasis and ivermectin treatment by the people in Okpuje, Edo State, Nigeria. International journal of Health Science.4 (3): 121 125 .

Rasheed MU., 2007. Onchocerciasis in Different Regions of Ethiopia. The Internet Journal of Parasitic Diseases.1:1.

Ukoli FMA., 1992. Prevention and control of parasitic diseases in tropical Africa: The main issues. Ibadan: University Press; 1992.

Wayuma T. A., 2015. Community-based cross-sectional study of the status of onchocerciasis, and awareness of the Community Drug Distributors (CDDs) about Onchocerciasis and Community Directed Treatment with Ivermectin (CDTI) in Homa District, Western Ethiopia.

World Health Organization, 2010. African program for onchocerciasis control: conceptual and operational framework of onchocerciasis elimination with ivermectin treatment, $J A F ; 16: 5-23$.

World Health Organization, 2000. Implementation and sustainability of community-directed treatment with ivermectin: report of a multi-country study. Geneva.

Workneh W., Fetcher M., Olwit G., 1993. Onchocerciasis in field workers at Baya farm, Teppi coffee plantation project, South Western Ethiopia: prevalence and impact on productivity Acta Trop 54: 89-99.

Yesuf A., 2006. Assessment of KAP of CDTI of onchocerciasis among the communities around Seqa area, southwestern Ethiopia. Addis Ababa, Ethiopia: MSC. Thesis, Addis Ababa University.

Yirga D, Woldemichael K, Wondafrash M, Kassahun W, Deribe K., 2008. Knowledge and Belief about Cause and Prevention of Onchocerciasis in Bebeka, Southwest Ethiopia. Ethiop J Health Sci: 18:66-68. 